\title{
Süßstoffe machen nicht schlanker
}

\section{Hier steht eine Anzeige.}

\section{Eine Metaanalyse findet keinen Nachweis, dass sich die Verwendung nicht-nutritiver Süßungsmittel positiv auf Gewicht und Gesundheit auswirkt.}
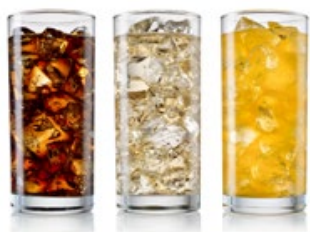

Light-Getränke für eine bewusste Ernährung?
- Die Analyse von sieben Interventionsstudien mit insgesamt 1.003 Teilnehmern und einer mittleren Beobachtungszeit von sechs Monaten ergab, dass nicht-nutritive Süßungsmittel keinen signifikanten Effekt auf den BMI haben. Daneben wurden 30 prospektive Kohortenstudien mit einer mittle-

ren Beobachtungszeit von zehn Jahren analysiert. Hier war regelmäßiger Süßsstoff-Konsum sogar mit einem moderaten BMI-Anstieg assoziiert, ferner mit einem erhöhten Körpergewicht und Taillenumfang sowie einem Inzidenzanstieg von Adipositas, Bluthochdruck, metabolischem Syndrom, Typ2-Diabetes und kardiovaskulären Ereignissen.

Die Autoren schlussfolgern, dass die randomisierten Studien keinen positiven Effekt nicht-nutritiver Süßungsmittel auf das Gewichtsmanagement belegen, auch wenn für eine eindeutige Aussage weitere und v. a. größere Studien nötig seien.

- Azad MB, Abou-Setta AM, Chauhan BF et al. Nonnutritive sweeteners and cardiometabolic health: a systematic review and meta-analysis of randomized controlled trials and prospective cohort studies. CMAJ. 2017;189:E929-39

\section{KOMMENTAR}

Die Übersichtsarbeit besticht durch die Berücksichtigung von randomisierten klinischen Studien, die als Goldstandard in der Beweisführung gelten, und prospektiven Beobachtungsstudien, mit denen Langzeiteffekte untersucht werden können. Für die klinische Relevanz sind primär die Ergebnisse der randomisierten klinischen Studien wichtig, die zeigen, dass Süßungsmittel keinen Nutzen für das Gewichtsmanagement haben. Allerdings ist die Anzahl der eingeschlossenen Studien und Studienteilnehmer sehr klein.

Bezüglich der Langzeiteffekte aus den Kohortenstudien ist darauf hinzuweisen, dass vielfach gezeigt wurde, dass gerade übergewichtige Personen regelmäßig kalorienfreien Süßungsmittel konsumieren.

Die Übersichtsarbeit lässt keine Aussagen bezüglich der DosisWirkungs-Beziehung zu. Auch unterscheidet sie nicht zwischen verschiedenen Süßungsmitteln. Sie stellt die aktuelle Datenlage sehr gut dar, lässt aber keine Ernährungs-Empfehlungen zu. Dafür wären groß angelegte Interventionsstudien mit einer mehrjährigen Nachbeobachtungszeit nötig. 